2020 TheoLogica

An International Journal for Philosophy of Religion and Philosophical Theology

S. I. CONCILIAR TRINITARIANISM

DOI: https://doi.org/10.14428/thl.v4i2.22183

\title{
La perichoresis e la grammatica teologica dei primi sette Concili ecumenici
}

\section{(Perichoresis: the Theological Grammar of the First Seven Ecumenical Councils)}

\author{
GIULO MASPERO \\ Pontificia Università della Santa Croce \\ maspero@pusc.it
}

\begin{abstract}
L'articolo analizza la storia del termine perichoresis nello spazio di tempo abbracciato dai primi sette concili ecumenici. Dopo l'esordio cristologico nel IV secolo della terminologia nell'opera di Gregorio di Nazianzo per indicare la dinamicità del rapporto delle due nature del Cristo nell'unione ipostatica, si mostra come questo passo teologico sarà la base dello sviluppo in Massimo il Confessore. Questi nel VII seccolo applicherà il guadagno teologico del Nazianzeno alla divinizzazione, esplicitando il fondamento cristologico della salvezza cristiana. Il percorso si conclude nell'VIII secolo con Giovanni Damasceno, il quale applica perichoresis sia alla cristologia, sia alla divinizzazione, come già prima di lui, ma estende la terminologia anche alla dimensione trinitaria, sigillando così la parabola del pensiero teologico. Ciò permette di riconoscere una vera e propria grammatica teologica che, coerentemente con gli studi di Timothy Pawl, rivela il valore architettonico di un Conciliar Trinitarianism, come epistemologia trinitaria che si fonda su un'antropologia trinitaria, a sua volta radicata in una ontologia trinitaria.
\end{abstract}

Keywords: Perichoresis, Gregorio di Nazianzo, Massimo il Confessore, Giovanni Damasceno, Conciliar Trinitarianism 


\begin{abstract}
The article analyzes the history of the term perichoresis in the space of time embraced by the first seven ecumenical councils. After the Christological debut of the terminology in the fourth century in the work of Gregory of Nazianzus to indicate the dynamism of the relationship of the two natures of Christ in the hypostatic union, the text shows how this theological transition was the basis of the development in Maximus the Confessor. In the seventh century he applied the theological gain of Gregory of Nazianzus to divinization, making explicit the Christological foundation of Christian salvation. The journey ends in the 8th century with John Damascene, who applies perichoresis to both Christology and divinization, as already seen before him, but extends the terminology to the Trinitarian dimension, thus sealing the parable of theological thought. This makes it possible to recognize a true theological grammar which, consistently with Timothy Pawl's studies, reveals the architectural value of a Conciliar Trinitarianism, as a Trinitarian epistemology based on a Trinitarian anthropology, in turn rooted in a Trinitarian ontology.
\end{abstract}

Keywords: Perichoresis, Gregory of Nazianzus, Maximus the Confessor, John of Damascus, Conciliar Trinitarianism

\title{
1. Introduzione
}

In Introduzione al Cristianesimo Joseph Ratzinger evidenzia un dato particolarmente interessante: tutti i termini fondamentali per il lavoro di formulazione del dogma trinitario che ha occupato il IV secolo dal Concilio di Nicea fino a Costantinopoli erano stati precedentemente condannati (Ratzinger 1971, 130). Prospon aveva un senso modalista, homoousios era stato utilizzato da Paolo di Samosata e i termini relativi alla processione erano comuni nell'ambito gnostico. Nella stessa formula fondamentale "una sostanza, tre ipostasi" il significato dei termini è determinato solo dalle relazioni, in quanto fino al III secolo i due sostantivi, distinti nell'espressione dai diversi numerali, erano considerati sinonimi (Ratzinger 1971, 135-7).

Ma allora è possibile parlare di indagine scientifica e di conoscenza di una verità nella ricerca storica? La ricostruzione è in qualche misura possibile oppure il senso rimane sempre al di là delle possibilità conoscitive dello studioso contemporaneo? L'approccio di Timothy Pawl si rivela a questo proposito particolarmente prezioso. Nel suo In Defence of Conciliar Christology (Pawl 2016) egli mette alla prova la coerenza filosofica della cristologia sviluppata nel corso dei primi secoli dell'era cristiana abbracciati dai primi sette concili ecumenici. Recentemente il valore filosofico della cristologia dei Padri della Chiesa è sempre più al centro dell'interesse (Zachhuber 2014 e 2015). Ma 
qui la questione è non solo storica, ma prima ancora epistemologica. Si tratta, infatti, di verificare se le relazioni interne all'insieme del dogma sviluppato in tale lasso di tempo possono fare emergere un significato più profondo rispetto a quello che le singole formule rivelano nella particolarità del loro contesto proprio.

La domanda è estremamente preziosa anche da un punto di vista metodologico, perché rende possibile mettere alla prova l'ipotesi che si possa applicare alla Dogmengeschichte quella correzione che l'esegesi ha apportato al riduzionismo storico-critico attraverso l'approccio canonico (Sanders 1984 e Childs 1979). Analogamente alla costatazione che il senso di un passo della Scrittura e il messaggio di un singolo libro che la compone possono essere colti solo collocando le diverse unità in quell'insieme che la tradizione ha tramandato come contesto proprio di esse, così ci si può domandare se i sette primi concili ecumenici offrano un quadro di riferimento efficace per cogliere i significati più profondi delle diverse affermazioni dogmatiche dei secoli da essi abbracciati. Lo studio della perichôresis in questo lasso di tempo si può rivelare particolarmente proficuo per tentare di rispondere a tale interrogativo, in quanto il suo sviluppo attraversa gli ambiti teologici principali, dalla cristologia, all'antropologia, fino alla dottrina trinitaria. ${ }^{1}$ In tal senso se ne può verificare la valenza architettonica nell'ambito di un Conciliar Trinitarism.

\section{I Cappadoci: l'incipit cristologico}

La presente ricerca seguirà principalmente il criterio terminologico, andando ad analizzare in primo luogo quei passi dove il termine perichôresis o il verbo ad esso legato fanno la loro comparsa. Per coglierne la valenza teologica, quando necessario, i testi verranno messi in parallelo con altri che paiono rilevanti per la loro interpretazione. L'indagine procederà senza pretesa di completezza alcuna, attraverso una dispositio pensata per evidenziare quella che Gregorio di Nissa chiamava akolouthia, cioè la successione temporale e la connessione essenziale dei significati teologici del termine.

La prima ricorrenza analizzata è, così, la più famosa. Gregorio di Nazianzo è, infatti, ritenuto l'autore che ha introdotto la terminologia in esame nell'ambito del pensiero cristiano, più precisamente in quello cristologico. Il passo è fondamentale per cogliere lo sviluppo della categoria nell'arco dei primi sette concili ecumenici:

Se qualcuno osasse dire che la carne [del Cristo] è scesa dal cielo e non è dalla terra e da noi, sia anatema. Infatti, le parole "il secondo uomo dal cielo" (1 Cor

1 Per una visione d'insieme, si vedano Stemmer 1983, Harrison 1991 e Mănăstireanu 20072008. 


\section{GIULO MASPERO}

15:47) e "ma quale il celeste, così anche i celesti" (1 Cor 15:48) e "nessuno è mai salito al cielo fuorché il Figlio dell'Uomo che è disceso dal cielo" (Gv 3:13) e le espressioni simili dovrebbero essere intese essere state pronunciate in riferimento all'unione ( $\check{v} v \omega \sigma \mathrm{v})$ [della natura umana] con quella celeste, cioé divina. Nello stesso senso, anche "tutto è stato fatto per mezzo" (Gv 1:3) di Cristo e Cristo abita nei nostri cuori (cfr. Ef 3:17) dovrebbero essere lette non in

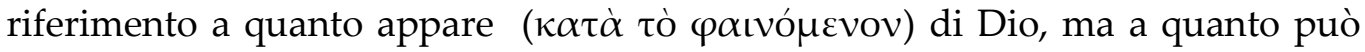

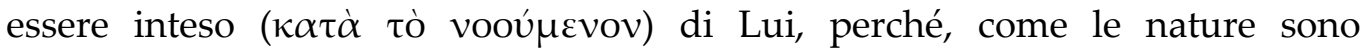

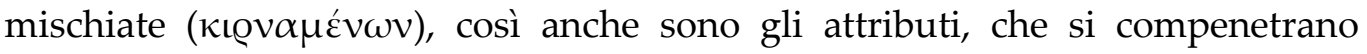

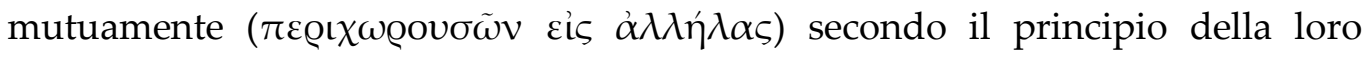

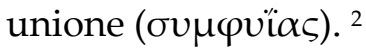

Si tratta del cuore stesso della teologia cappadoce, impegnata nella risposta al subordinazionismo di Eunomio e, nel caso dei due Gregori, anche al riduzionismo cristologico e antropologico di Apollinare. Qui il Nazianzeno sta sviluppando la distinzione tra economia e immanenza, la cui origine è esegetica. Il punto è quali testi vadano letti in riferimento all'umanità di Cristo e quali alla Sua divinità. Basilio aveva impostato la questione nel suo commento ad At 2:36. ${ }^{3}$ Il Nisseno l'aveva ripresa. ${ }^{4}$

Nel passo di Gregorio di Nazianzo appena citato l'interpretazione di 1 Cor 15 è collocata sullo sfondo della netta distinzione delle due nature del Cristo, la cui unione, però, è intesa dinamicamente. Ciò è espresso attraverso l'applicazione del verbo perichôreô all'ontologia di Gesù di Nazareth. La sequenza teologica è estremamente chiara e precisa. In primo luogo (a) si distingue la dimensione del katà tò phainómenon da quella del katà tò nooúmenon, per poi (b) evidenziare come tale distinzione sussista in un'unione che non si dà solo al livello delle proprietà, ma tocca la profondità metafisica delle nature, in modo tale che (c) la communicatio idiomatum è riflesso della dinamicità dell'unione che è formulata proprio con il verbo da cui deriva perichôresis.

Nell'insieme delle opere del Nazianzeno, lo stesso verbo è impiegato nell'Oratio XXII (De pace) in un contesto negativo, per descrivere come la discordia si diffonda anche tra i cristiani, perché anche se si è "pieni di tutto, sia di ciò che fa soffrire, sia di ciò che è più dolce, tutto volge su di sé e ritorna

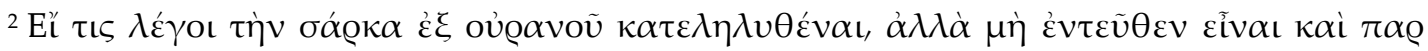

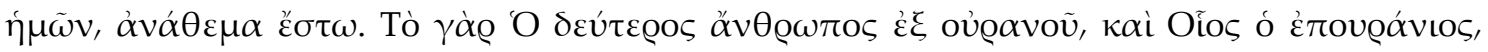

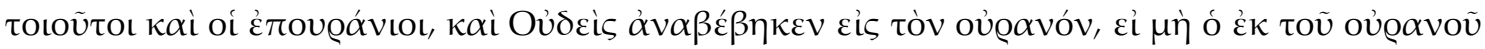

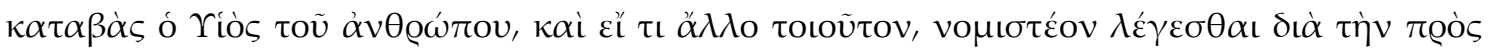

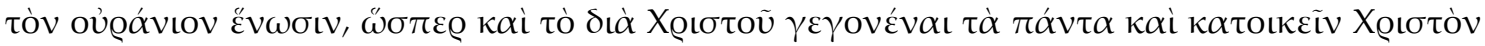

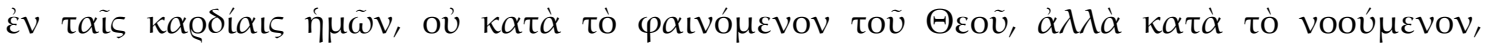

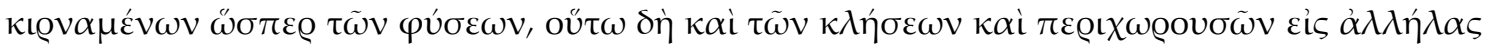

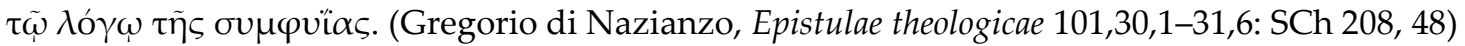

${ }^{3}$ Cf. Basilio, Adversus Eunomium, PG 29, 577A.

${ }^{4}$ Cf. Gregorio di Nissa, Contra Eunomium III, 3, 12, 38; GNO II, 111, 1419. 


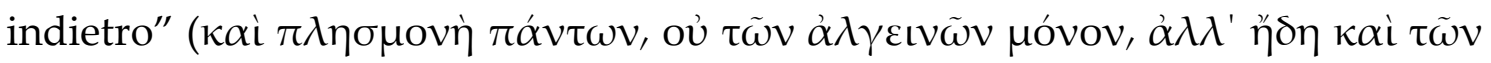

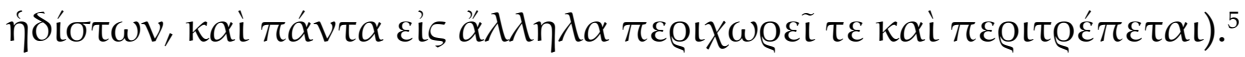

La terza occorrenza può aiutare a cogliere il senso e la connessione dei due primi passi citati, perché ne è quasi una sintesi. Infatti, verso la fine dell'Oratio XVIII, discorso funebre per il proprio padre, il Nazianzeno consola la madre Nonna evidenziando la differenza tra la natura divina e quella umana:

O Madre, la natura di Dio e degli uomini non è la stessa, anzi, più in generale, non è la stessa quella delle realtà divine e quella delle realtà terrene. Le prime sono caratterizzate dall'immutabilità e dall'immortalità, sia per quanto riguarda l'essere sia per quanto riguarda ciò che all'essere corrisponde. Infatti, ciò che è stabile appartiene alle realtà stabili. Invece come si comporta $(\pi \tilde{\omega} \varsigma$ É $\chi \varepsilon \iota)$ la nostra natura? Scorre e si corrompe e si trasforma ora in un modo ora in un altro. ${ }^{6}$ Perfino la vita e la morte, che come si dice presentano la più grande differenza reciproca, in un certo modo si compenetrano mutuamente ( $\varepsilon i \varsigma$ $\not \check{\alpha} \lambda \lambda \eta \lambda \alpha \pi \varepsilon \varrho \iota \chi \omega \varrho \varepsilon \tilde{)})$ e si intercambiano. ${ }^{7}$

Senza entrare nella discussione tra la Harrison e Prestige sul senso dell'ultima espressione (Harrison 1991 e Prestige 1928), si può, dunque, dire a grandi linee che il verbo serve a Gregorio di Nazianzo per esprimere la mutabilità intrinseca alla contingenza creaturale. Il punto fondamentale, però, è che tale dinamicità apre cristianamente la prospettiva soteriologica. Il Cappadoce, infatti, sta consolando la propria madre per la morte del proprio padre. Addirittura, nel seguito del testo, arriva a spiegarle con forza paradossale che la vita stessa inizia dalla corruzione, che è "madre" degli uomini, ${ }^{8}$ per culminare nella morte, dopo aver attraversato tutta la corruzione dell'esistenza mortale. Ma, da tale prospettiva, i ruoli della vita e della morte si scambiano, perché quest'ultima apre alla vera vita, cioè a quella dimensione divina di cui parla il testo citato. La dinamica è molto evidente nell'attenzione lessicale: la morte non dovrebbe

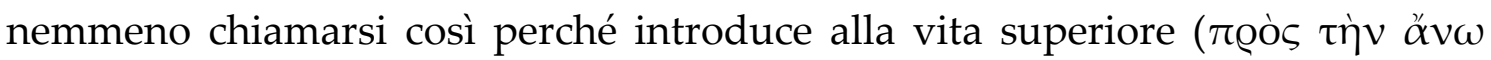
$\zeta \omega \eta ́ v)$. Per questo non dovrebbe essere temuta. Da qui deriva la forza consolatoria del testo, perché "una sola è la vita: rivolgersi alla vita. Una sola la

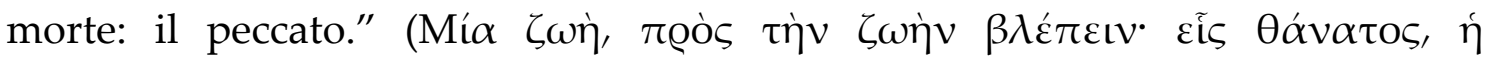

\footnotetext{
${ }^{5}$ Gregorio di Nazianzo, Oratio 22,4: SCh 270, 226.

${ }^{6}$ Claudio Moreschini riviene qui un richiamo eraclideo (Moreschini 2000, 1276).

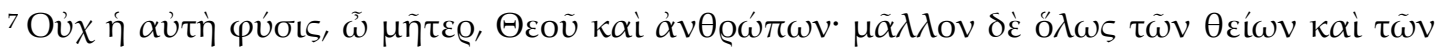

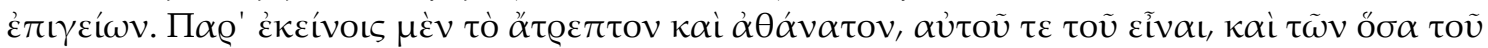

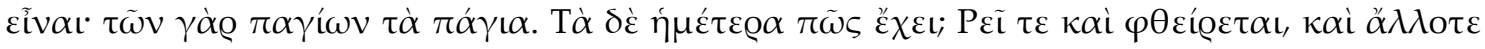

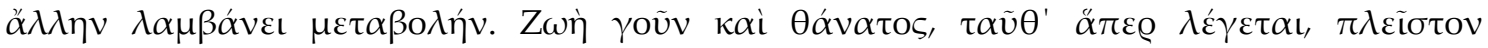

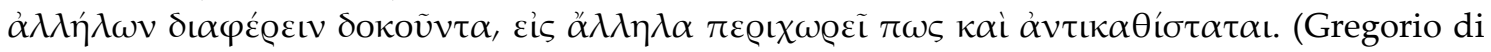
Nazianzo, Oratio 18,42: PG 35, 1040C-1041A)

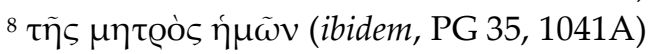




\section{GIULO MASPERO}

$\alpha \mu \alpha \varrho \tau i ́ \alpha){ }^{9}$

Si vede, dunque, fin dal primo utilizzo teologico, che la connessione tra la dimensione cristologica e quella soteriologica è al centro delle scelte del Nazianzeno. La vita e la morte in realtà dovrebbero scambiarsi le posizioni, alla luce della fede, perché in Cristo la natura dell'uomo è unita una volta per sempre alla natura divina, che è la Vita, senza limiti. In Lui la mutevolezza dell'uomo, i suoi limiti, diventano benedizione, perché permettono di accogliere l'infinito nel finito. Il tema è fondamentale anche nel pensiero del Nisseno, il quale parla proprio del valore positivo del movimento. ${ }^{10}$

Per questo sembra significativo leggere i passi del Nazianzeno in relazione proprio con il pensiero dell'altro Gregorio, la cui teologia è stata estensivamente studiata in relazione alla perichôresis (Stramara 1998). Anche lui distingue tra katà tò phainómenon e katà tò nooúmenon, lavorando nello stesso contesto esegetico e cristologico. Classiche sono le critiche presenti in letteratura di ambito sistematico al suo uso della terminologia legata alla mixis, così come all'immagine della goccia di aceto versata nel mare, per spiegare l'unione delle due nature nel Cristo (Tixeront 1909, 128-30 e Grillmeier 1990, 540-1). Le ricostruzioni da parte patristica hanno, però, messo in luce il vero significato delle espressioni cappadoci, superando l'anacronismo dei giudizi precedenti (Bouchet 1968 e Mateo-Seco 2003). ${ }^{11}$ Infatti, il II Concilio di Costantinopoli fece ricorso proprio alla teologia Cappadoce per interpretare in senso realista Cirillo, evidenziando che dopo l'unione la distinzione delle nature è reale, e non solo logica, ma indissolubile, in modo tale che solo il pensiero può riconoscere le caratteristiche di ciascuna nell'unità dell'ipostasi (Maspero 2004). I teologi di Giustiniano fecero ricorso proprio ai Cappadoci per evidenziare l'interconnessione dogmatica della distinzione delle nature nel Cristo e la distinzione delle Persone divine nella Trinità. ${ }^{12}$ L'espressione usata dal Concilio è theôria monê, che si può ricollegare al katà tò nooúmenon sopracitato. Da tale prospettiva assume un valore particolare il seguente testo:

E Egli [il Figlio] è sempre nel Padre ed ha sempre il Padre in Sé stesso ed è una cosa sola con Lui, come è stato ed è e sempre sarà; e un altro Figlio che non sia

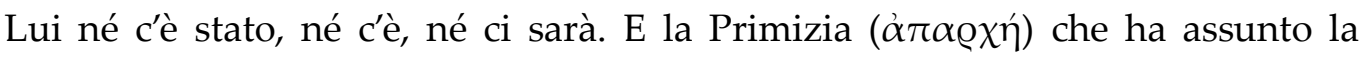
natura umana è nella divintà e non solo nelle caratteristiche ad essa proprie,

\footnotetext{
${ }^{9}$ Cf. ibidem.

${ }^{10}$ Cf. Gregorio di Nissa, De anima, PG 46, 157B.

${ }^{11}$ Più recentemente, si vede il magnifico lavoro di Brian Daley God Visible: Patristic Christology Reconsidered (Daley 2018).

${ }^{12}$ Ad esempio, nel Edictum rectae fidei si citano di Gregorio di Nissa il Contra Eunomium III, 4, 14,6 -15,12 (GNO II, 138,28-139,17) in Schwartz, 142,37-144,10 e Refutatio confessionis Eunomii, 12,4-13,3 (GNO II, 317, 20-27) in Schwartz, 70, 3-7, testi che collegano l'unione nel Cristo e quella trinitaria delle Persone divine.
} 
come una goccia $(\sigma \tau \alpha \gamma \omega \dot{\omega} v)$ di aceto mischiata dall'onnipotenza divina con un

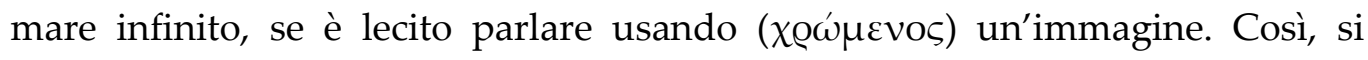
potrebbe pensare coerentemente a una diade di figli, se si potesse riconoscere una natura eterogenea attraverso proprietà specifiche nell'ineffabile divinità del Figlio, in modo tale che Egli sarebbe, da un parte, debole, piccolo, mortale e temporale, ma potente, grande, immortale ed eterno, dall'altra. Ma, poiché tutte

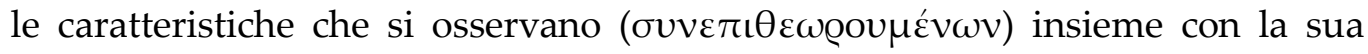
mortalità sono trasformate nelle proprietà della sua divinità, non si può cogliere differenza alcuna, poiché tutto ciò che può essere visto nel Figlio è divinità, Sapienza, potenza, santità e impassibilità, come potrebbe essere possibile dividere l'unica realtà in una duplice indicazione, quando nessuna differenza divide il numero? ${ }^{13}$

L'importanza del testo, nel presente percorso, può essere riconosciuta nel collegamento tra ciò che Gregorio di Nazianzo esprime attraverso il verbo perichôreô a livello cristologico e una chiara espressione da parte di Gregorio di Nissa di quella mutua inabitazione delle Persone divine l'una nell'altra che poi diventerà il senso classico della perichôresis trinitaria. Tale movimento ideale dei due Cappadoci si fonda sulla dimensione soteriologica cui rinvia la communicatio idiomatum a livello ontologico. La domanda che guida la nostra ricerca è: come questo nucleo è stato sviluppato nella teologia patristica legata ai primi sette concili ecumenici? Il riferimento a Constantinopoli II è solo casuale oppure si ha una sorta di interconnessione, quasi di "pericoresi" a livello di contenuti dogmatici? E tale sviluppo presenta una coerenza o l'uso (chrêsis) delle diverse espressioni è meramente formale e funzionale?

\section{Massimo il Confessore: lo sviluppo soteriologico}

Massimo il Confessore fu attento lettore degli scritti del Nazianzeno, dal quale mutuò e incrementò l'uso della terminologia relativa alla perichôresis (Harrison 1991, 56-7). Se veramente gli Scholia dell'Areopagita sono stati raccolti da

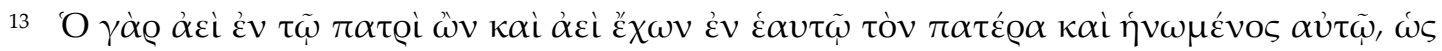

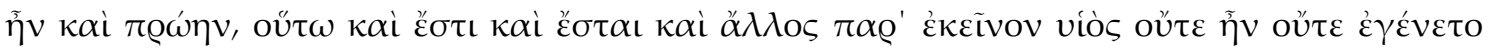

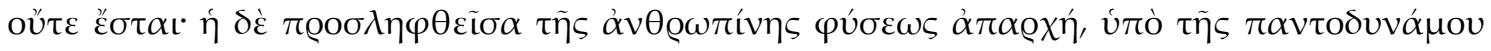

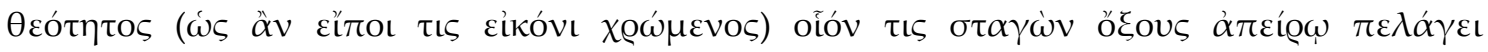

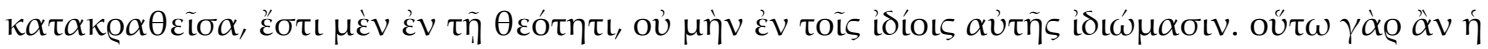

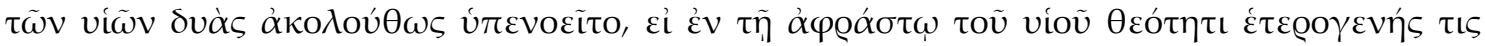

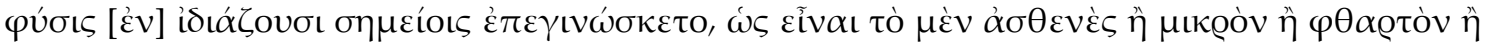

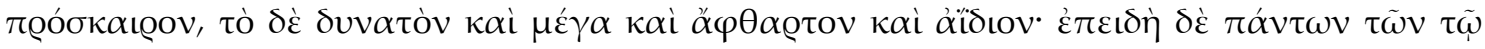

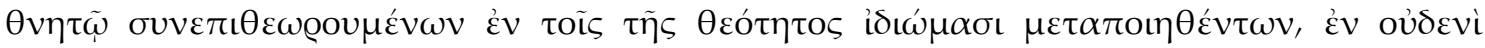

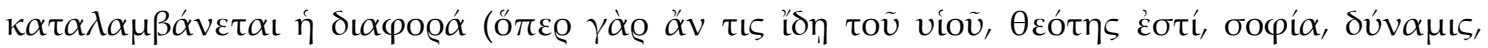

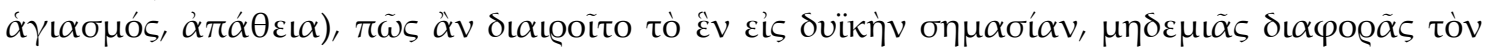

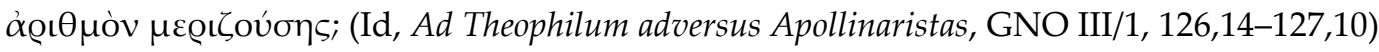




\section{GIULO MASPERO}

Massimo, troviamo un'esplicita citazione del primo testo proposto all'inizio della nostra indagine. Esso si colloca ovviamente in ambito cristologico, dove viene spiegato che Gregorio di Nazianzo (Gregorio teologo) introdusse la terminologia legata a perichôresis per indicare l'ammirabile e inspiegabile unione di divino e umano in Cristo. In base ad essa si può dire di Lui che Dio rese solido il mare ma l'uomo vi camminò sopra su piedi di carne, e che Dio fece partorire la Vergine ma l'uomo ne ebbe volto e membra ${ }^{14}$.

Probabile pare anche il riferimento al testo del Nazianzeno nell' Ad Marinum, dove la perichôresis appare insieme alla symphyia e al pros allêlas:

Quindi, mostrando le operazioni naturali di lui stesso che è il Cristo Dio, unione di entrambe, preservate perfettamente, quella della sua divinità mediante la sua potestà di efficacia universale, quella della sua umanità attraverso il tatto, le presentò completamente unite nella reciproca congiunzione e compenetrazione

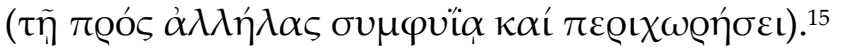

Il vocabolario e la teologia puntano evidentemente alla teologia cappadoce e specificamente al testo della Ep 101 del Naziazeno dove il verbo tratto dalla stessa radice di perichôresis è introdotto per la prima volta in ambito cristologico. Da subito emerge l'attenzione di Massimo alle operazioni del Cristo, per il mutato contesto teologico in cui scrive. Così, riprendendo il filo, si potrebbe dire che la distinzione nella compenetrazione delle due nature, riflessa nella distinzione nella compenetrazione delle proprietà, già evidenziate da Gregorio, viene ora estesa alle proprietà naturali del Cristo.

Possiamo così vedere nuovamente l'interconnessione tra il vocabolario della perichôresis e l'esemplificazione esegetica della comunicatio idiomatum, questa volta, però, nell'intento di mostrare come le proprietà rimangano distintamente attribuibili alle due diverse nature, senza che tale distinzione venga meno nella considerazione dell'unità dell'operazione operata dal Cristo.

Lo stesso si ripete più oltre nello stesso Ad Marinum. Infatti, mentre mostra che in un'azione attribuita al Maestro vi è l'espressione della potenza di due principi distinti, che sono le nature divina e umana, il Confessore mette in evidenza altresì che l'effetto è uno solo, indivisibile e non moltiplicabile; si può parlare pertanto di un'unica operazione nella quale agiscono le due nature, distintamente e insieme, secondo una singola e unitaria mutua immanenza

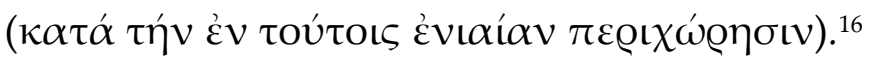

${ }^{14}$ Cf. PG 4, 533C. Si veda in proposito CPG 7708 (p. 446).

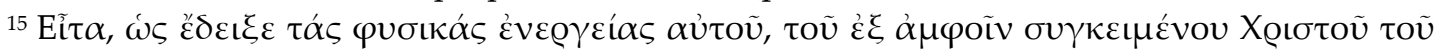

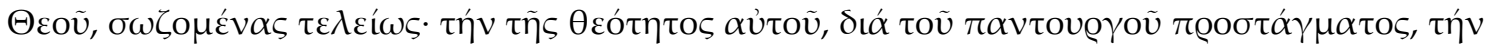

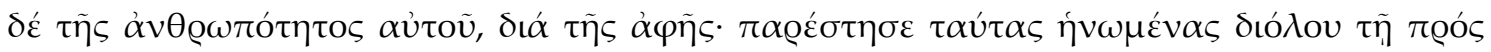

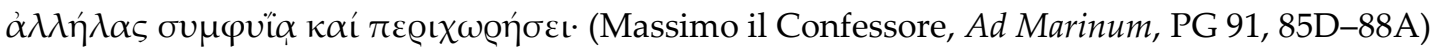

${ }^{16}$ Cf. ibidem, PG 91, 232AB. 
Tale impostazione evidenzia la prospettiva soteriologica. Così, in Capitum Quinquies Centenorum Centuria, IV si rinviene un passaggio-che si ripete identico nella questione Ad Thalassium 59 (cfr. SCh 569, p. 64)-dove Massimo mostra un uso particolarmente importante per intendere la rilevanza della perichôresis per la salvezza cristiana assunta nel dibattito post-calcedoniano. Egli presenta una vertiginosa articolazione teologica del cammino verso la divinizzazione. Centro del suo interesse è il rapporto grazia-natura, che egli intende in senso relazionale piuttosto che dialettico. Lo Spirito Santo si appoggia, infatti, alle facoltà naturali: ${ }^{17}$ la conoscenza spirituale sarebbe impossibile senza la presenza della terza Persona, come l'occhio umano non potrebbe vedere nulla senza la luce del sole. È il dono divino che permette alla facoltà di percepire l'oggetto conosciuto che di per sé è al di là delle sue possibilità. Quindi Massimo introduce la citazione di 1 Pt 1,9:

In senso proprio "la salvezza delle anime è il fine della fede" (1 Pt 1,9). Il fine della fede è la rivelazione veritiera di Colui in cui si ha fede. Ma la rivelazione

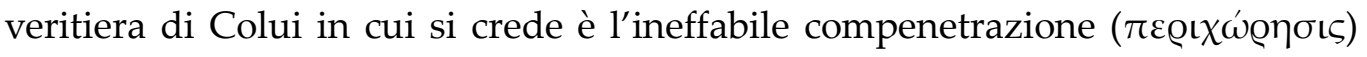
con Colui in cui si crede in proporzione alla fede di ciascuno. E la compenetrazione ( $\pi \varepsilon \varrho \iota \omega \omega \emptyset \eta \sigma \varsigma)$ con Colui in cui si crede è il ritorno definitivo al principio proprio di coloro che credono. E il ritorno al principio proprio di coloro che credono è il compimento del desiderio ( $\dot{\varepsilon} \varphi \dot{\varepsilon} \sigma \varepsilon \omega \varsigma)$. E il compimento del desiderio è il riposo sempre in movimento nell'oggetto del desiderio da parte di coloro che lo desiderano. E il riposo sempre in movimento è il godimento continuo e senza limite dell'oggetto desiderato. Ma il godimento continuo e senza limite è la partecipazione $(\mu \dot{\varepsilon} \theta \varepsilon \xi \iota \varsigma)$ alle realtà divine che superano la natura. E la partecipazione alle realtà divine che superano la natura è la somiglianza (ómoí $\omega \sigma \iota \varsigma)$ dei partecipanti rispetto al partecipato. E la somiglianza dei partecipanti rispetto al partecipato è l'identità secondo l'atto dei partecipanti rispetto allo stesso partecipato ricevuto attraverso la somiglianza. E l'identità secondo l'atto dei partecipanti rispetto allo stesso partecipato ricevuto attraverso la somiglianza è la divinizzazione $(\theta \varepsilon \dot{c} \omega \sigma \iota)$ di coloro che sono degni della divinizzazione. ${ }^{18}$

${ }^{17}$ Cf. Massimo il Confessore, Ad Thalassium 59, 89-116: SCh 569, 62-4.

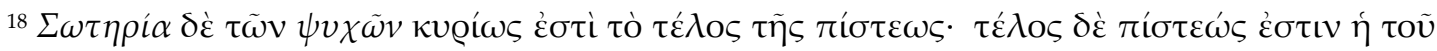

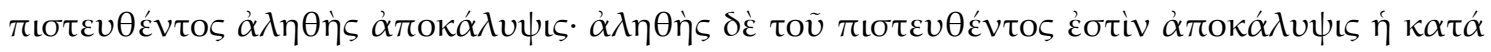

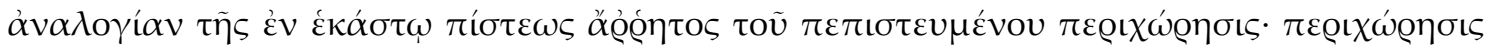

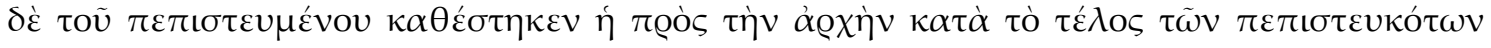

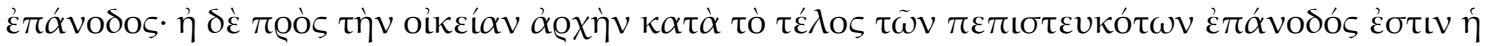

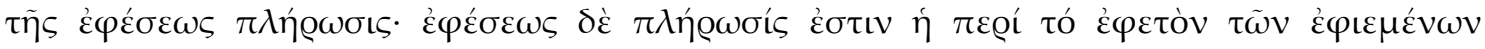

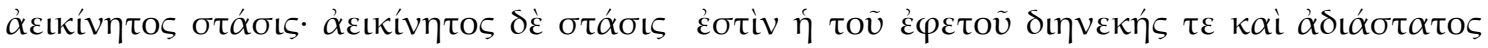

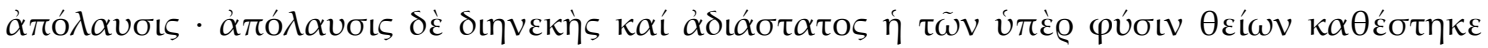

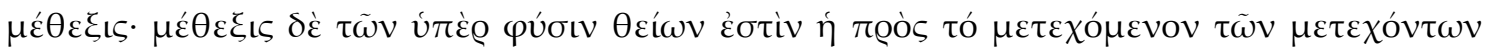

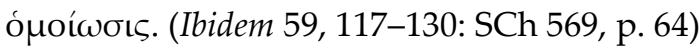




\section{GIULO MASPERO}

In questo testo vediamo come, mediante la fede, la sequenza antropologica che porta dalla pienezza del desiderio alla partecipazione alla vita divina e dalla somiglianza con Dio alla piena divinizzazione dell'essere umano passi, secondo Massimo, attraverso l'interpenetrazione dell'agire, dell'uomo con Dio, dell'uomo e di Dio. ${ }^{19}$ Il volere ha, qui, una profonda dimensione ontologica, per la quale si diventa quello che si vuole, fino all'estremo di poter essere divini nell'unione con Cristo. La soteriologia apre, così, il cammino alla mistica. La priorità dell'azione divina è chiara ed è data dall'economia salvifica, ma la relazione con la persona umana sottolineata dal ricorso alla perichôresis appare evidente proprio per la distinzione che il termine stesso suppone e esprime tra $\mathrm{i}$ soggetti messi in comunione vitale.

Verna Harrison sottolinea la differenza tra la divinizzazione raggiunta dall'umanità di Cristo e la divinizzazione possibile per i santi, dovuta alla unicità dell'unione ipostatica nella quale l'azione delle nature inerisce perfettamente l'una nell'altra. Senza entrare ancora nella discussione, importa qui nuovamente sottolineare è come l'uso di perichôresis possa da una parte avvalersi della distinzione nella unione delle nature-già stabilita chiaramente dal Concilio di Calcedonia e che mantiene invariata l'impassibilità divina nell'unione con l'umano-e dall'altra consenta di sviluppare la dimensione personale della relazione salvifica e della reciprocità dell'essere umano con l'azione del divino.

Infatti, la catena di identità che sorprendentemente si succedono nel testo di Massimo implicano che il fine della fede coincida con la perichôresis di Dio e dell'uomo per la fede la quale a sua volta coincide con l'homoiôsis e con la theôsis. È evidente che il significato di tale sequenza è quello della piena compenetrazione di divino e umano che permette alla natura creata di accedere alla vita imperitura del Creatore. Possiamo quindi rinvenire in questo brano la radice cristologica nella sua espressione più genuinamente soteriologica e mistica. Ciò è confermato dal ricorso a perichôresis nella disputa con Pirro e dalla sua prospettiva escatologica. La mutua e reciproca azione delle nature in Cristo diviene, infatti, il segno del modo di essere della nuova creazione. La novità, spiega Massimo, non consisterà tanto nella possibilità di compiere nuove operazioni, bensì in un nuovo modus operandi, quello inaudito della piena comunione di umano e divino, così come si è data nel Verbo incarnato:

Quando l'Apostolo dice "Ecco, diventano nuove tutte le cose" (2 Cor 5,17), egli null'altro dice se non che "Ecco, tutte le cose diventano una sola cosa". Sia che

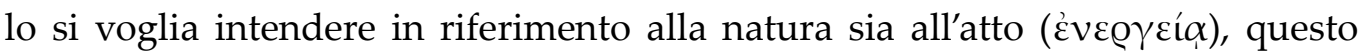

${ }^{19}$ Brian T. Scalise si sofferma a notare che tale interpenetrazione ha un modo di darsi personale, denotato dall'uso del pros e che indica contestualmente il riferimento al pros ton theon di Gv 1,1 (Scalise 2012, 66). 
deve essere riferito alla nostra potestà. Ma se la novità è qualitativa, non indica un solo atto ( $\dot{v} \mathcal{E} @ \gamma \varepsilon\llcorner\alpha v)$, ma sia il modo nuovo ed ineffabile di manifestazione delle operazioni naturali del Cristo, che corrisponde all'ineffabile forma della mutua compenetrazione ( $\pi \varepsilon \varrho \iota \chi \omega \emptyset \eta ́ \sigma \varepsilon \omega \varsigma)$ delle nature del Cristo, sia quel genere di vita secondo la sua umanità, che essendo straordinaria e paradossale, è ignoto agli esseri naturali, e indica la forma dello scambio secondo l'unione

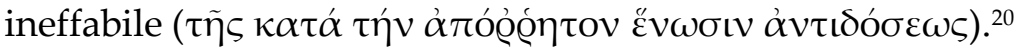

L'attenzione di Massimo è certamente rivolta alle operazioni del Cristo nel rapporto con le due nature, cioè proprio a quel luogo dogmatico dove soteriologia e cristologia si raccordano.

Se volessimo sottolineare l'eredità cappadoce presente in questo testo, dovremmo fare riferimento alla connessione tra economia salvifica ed immanenza divina, corrispondenza tra essere e agire di Dio che viene difesa non soltanto nella discussione con Ario ed Eunomio, ma anche in quella con Apollinare sull'unità del Verbo incarnato.

Il nesso tra l'essere e il modo di agire di Cristo è esplicitato in modo eminente nel Liber Ambiguorum dove Massimo spiega che Cristo è pienamente uomo ma ha un modo di essere uomo che supera la natura umana. Egli infatti si è unito liberamente alla natura umana e dunque la possiede non secondo la natura umana ma per assunzione, ovvero prendendola dal di sopra della natura umana stessa. L'ipostasi del Verbo, dunque, fa sì che la natura umana si unisca in modo inconfuso alla natura divina, la quale prende in sé, totalmente e in maniera che non sia mai più divisa dall'eterno e immutabile, la natura mortale e corruttibile.

Il Confessore sta parlando della Theotokos, commentando il suo essere Vergine e Madre, realtà contraddittorie a livello naturale. Per questo riafferma che l'unità del Cristo $d a$ e in due nature richiede il permanere dell'operazione insieme alla natura che la rende possibile. ${ }^{21}$

“E lui compiva le azioni umane ( $\tau \alpha \dot{\alpha} \alpha \nu \theta \varrho \omega ́ \tau o v)$ in una forma superiore a quella umana", secondo un'unione somma che senza implicare cambiamento mostra la congiunzione dell'operazione umana con la

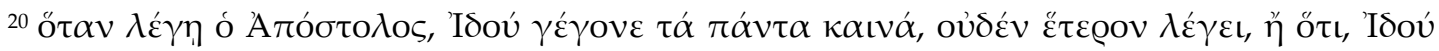

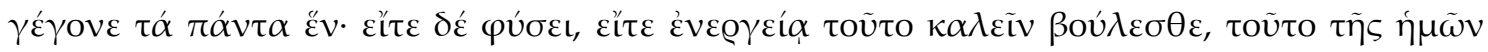

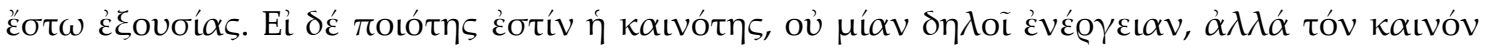

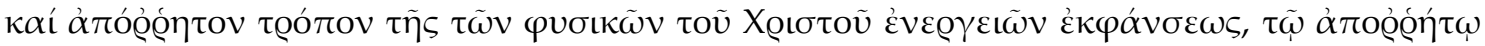

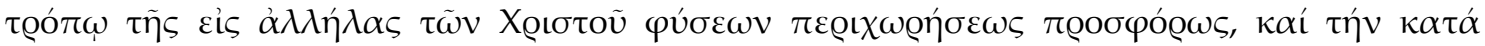

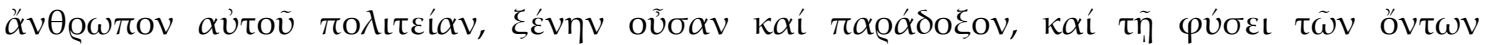

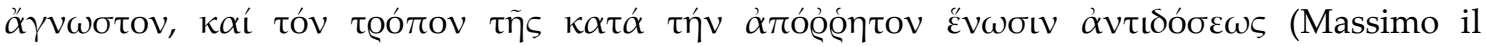
Confessore, Disputatio cum Pyrrho, PG 91, 345D-348A)

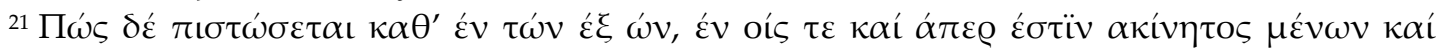

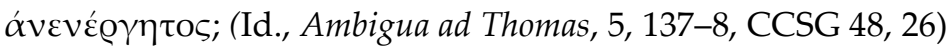




\section{GIULO MASPERO}

potenza divina. Infatti la natura [dell'uomo], unita senza confusione con quella divina, è stata tutta compenetrata ( $\pi \varepsilon \varrho \iota \kappa \varepsilon \chi \omega ́ \emptyset\rceil \varepsilon)$ da essa, senza che assolutamente nulla di essa fosse separata dalla divinità alla quale è stata unita secondo l'ipostasi. Infatti, "il Verbo che è sovraessenziale si è veramente unito in una forma superiore a noi" alla nostra sostanza e, divenuto uomo, ha unito l'apofasi sublime all'affermazione della nostra natura e delle sue proprietà naturali. Ha unito, così, il suo modo di

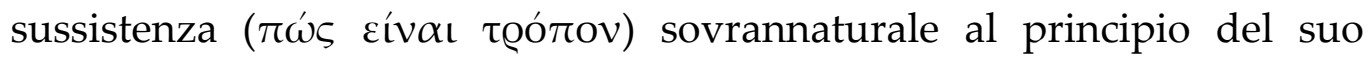
essere naturale, affinché anche la natura fosse confermata dalla novità dei modi senza alcuna mutazione nel principio, mostrando così la potenza sovraeminente riconosciuta nella generazione degli opposti ( $\tau \omega \dot{v}$

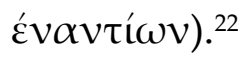

In questo testo si evidenzia la novità ontologica della teologia di Massimo, e come l'incarnazione avvenga nella piena libertà dell'ipostasi divina rispetto alla natura creata a cui si unisce.

Nell' Ambiguorum Liber stesso l'orizzonte distintivo nell'uso di perichôresis, pur continuando a rimanere su uno sfondo stoico, presenta una radicale novità di significato. Massimo indica, infatti, che, come nel cosmo, anche nel corpo umano gli opposti sono preservati uno nell'altro. Nel caso della natura,

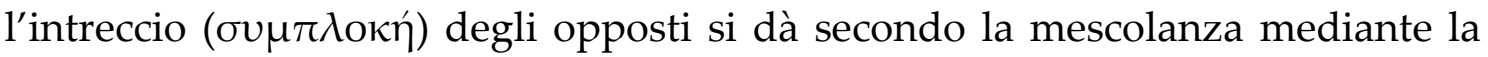

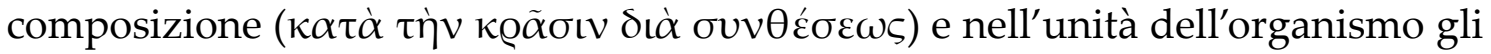

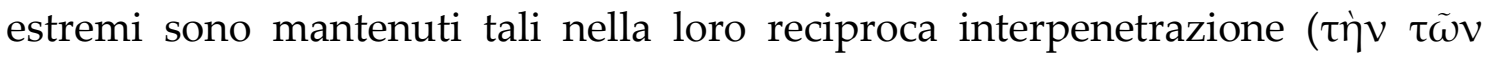

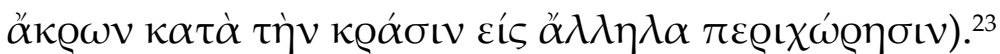

Si nota così lo sforzo proprio di Massimo di mantenere al contempo la distinzione e il parallelismo tra l'unione dinamica della dimensione naturale e soprannaturale, nella logica della salvezza, e la composizione unitaria degli elementi opposti del mondo creato, nella logica della creazione.

A sintesi di questa breve carrellata dei luoghi teologici più significativi, a riguardo delle ricorrenze di perichôresis nell'opera di Massimo il Confessore, possiamo dire che l'unione delle nature in Cristo è ripresa e approfondita nella

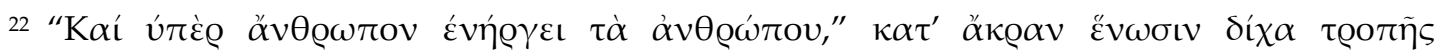

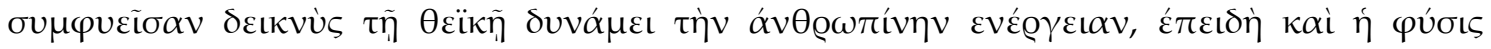

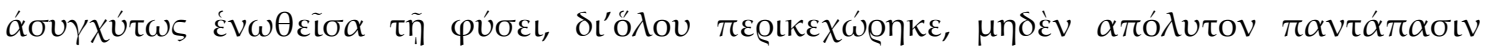

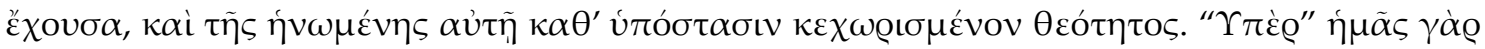

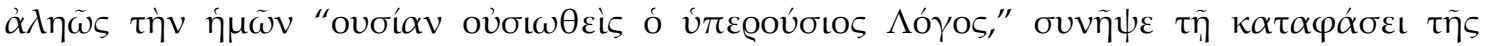

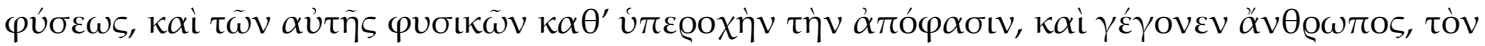

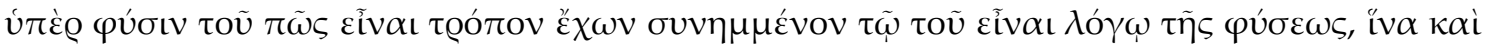

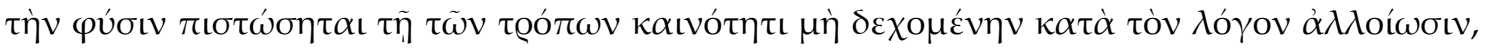

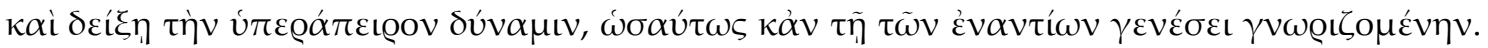
(Ibidem, 5, 150-162, CCSG 48, 26-27)

${ }^{23}$ Cfr. Id., Ambiguorum Liber, PG 91, 1228CD. 
linea della unità di effetto a partire dalle operazioni attribuibili distintamente alle due nature, secondo le quali le azioni vengono operate; mentre infatti si riscontra ancora il linguaggio stoico della mescolanza, si può notare che nell'ambito dialogico ormai decisamente post-calcedoniano esso ha perso ogni ambiguità e può essere impiegato per riaccostare i lembi della spaccatura ecclesiale tra difisiti e monofisiti. L'unità dell'effetto operativo delle azioni teandriche di Cristo è descritta mediante il ricorso efficace della terminologia della perichôresis, non soltanto per riaffermare la presenza del doppio principio operativo, ma anche per mostrare la superiorità di Cristo rispetto alle leggi naturali, perché il Lui la natura è assunta, non ricevuta. Ne consegue che l'antropologia escatologica che emerge dalla cristologia della perichôresis lascia intendere un avanzamento nella concezione del destino proprio della divinizzazione della natura umana e della nuova creazione che avrà compimento alla fine dei tempi: in tale condizione finale, secondo Massimo non avremo accesso ad operazioni diverse da quelle proprie della natura umana, ma sarà diverso il modo di agire, non più da sotto la legge della necessità naturale, ma dall'alto della libertà e dell'amore che viene dalla vita divina.

\section{Giovani Damasceno: Il compimento trinitario}

Spostandoci all'VIII secolo, subito prima della fine del percorso conciliare che culmina con Nicea II, Giovanni Damasceno offre un contributo fondamentale per la configurazione della categoria teologica della perichôresis. Basterebbe tale elemento a mettere in crisi ogni descrizione di questo Padre della Chiesa come mero compilatore. Egli scrive, infatti, per una comunità perseguitata, che è passata ad essere minoranza, e, tra le mille difficoltà, cerca di offrire ai cristiani una sintesi profonda che permetta di vivere la propria fede in un periodo così complesso (Louth 1996 e Twombly 2015). Il confronto con l'Islam rende evidentemente necessaria una formulazione sempre più chiara dell'unità e della trinità del Padre, del Figlio e dello Spirito che metta i cristiani al riparo da equivoci mortali. La categoria in esame passa, così, naturalmente al centro della costruzione dogmatica, per rendere ragione del:

reciproco stare ed essere fondate delle ipostasi l'una nell'altra. Infatti, esse sono senza intervallo e mutuamente inseparabili poiché posseggono la pericoresi di una nell'altra senza confusione ( $\alpha \sigma u ́ \gamma \chi v \tau o v ~ \pi \varepsilon \varrho \iota \chi \omega ́ \emptyset \sigma \iota v)$, non in modo da saldarsi e confondersi, ma possendendosi l'una con l'altra. Il Figlio è nel Padre e nello Spirito, lo Spirito è nel Padre e nel Figlio, il Padre è nel Figlio e nello Spirito, senza fusione alcuna o unificazione o confusione. Poiché unico è lo 


\section{GIULO MASPERO}

spazio e il movimento delle tre ipostasi, che è impossibile osservare $(\theta \varepsilon \omega \varrho \eta \theta \tilde{\eta} v \alpha \iota)$ nella natura creata. ${ }^{24}$

La perfezione e la compiutezza della formulazione sono luminose. Il termine perichôresis è associato all'aggettivo asynchytos, che già nei Cappadoci appariva sia in ambito trinitario che cristologico, quest'ultimo consacrato dal Concilio di Calcedonia. Come già in Gregorio di Nazianzo, l'intento del Damasceno è quello di evidenziare la differenza della natura divina rispetto alla creazione. La concezione delle mutue relazioni tra le Persone divine non è statica, come si darebbe in una miscela, bensì dinamica, secondo una prospettiva ontologica che è del tutto sconosciuta a livello diastematico-per usare un'espressione di Gregorio di Nissa relativa alla dimensione categoriale del mondo. Tale aspetto della realtà trinitaria trova però nella storia del mondo un suo corrispettivo a livello cristologico. Giovanni nota, infatti, che se Cristo fosse diventato una nuova terza natura composta a partire da quella umana e divina, Egli non sarebbe stato più consustanziale né a noi, cioè né a sua madre, né a Dio, tanto da non poter più essere chiamato né uomo né Dio, ma soltanto Cristo, dove quest'ultimo termine non sarebbe più il nome dell'ipostasi ma della nuova natura così emersa. Invece, secondo la dottrina ortodossa, il Cristo è $d a$ ed in

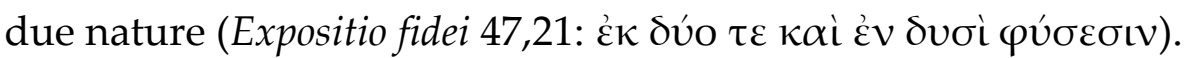

Si può qui vedere come la correlatività delle formulazioni dogmatiche sia fondata nell'eccedenza della profondità del mistero rispetto alle capacità conoscitive dell'uomo. Come pozzi diversi che attingono ad un'unica sorgente, la definizione dell'unità delle Persone divine è inseparabile rispetto a quella dell'unità delle nature del Cristo. Secondo lo stesso movimento interpretativo inaugurato da Giustiniano nel II Concilio di Costantinopoli, l'unità nella distinzione cristologica è fondata in quella trinitaria. Il Damasceno chiarisce che l'unione delle due nature del Cristo è, infatti, reale e non meramente logica, ${ }^{25}$ così come le tre Persone divine sono realmente distinte pur essendo un'unica infinita ed eterna sostanza. Nell'uso cristologico si nota immediatamente anche la connessione con la dimensione soteriologica, già presente fin dagli esordi degli usi della terminologia in Gregorio di Nazianzo:

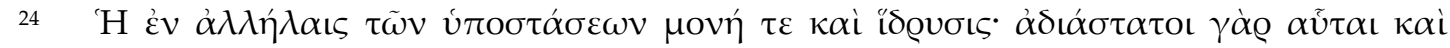

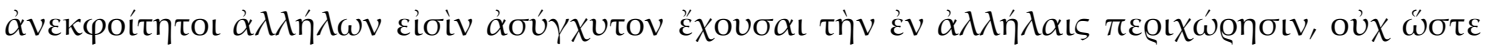

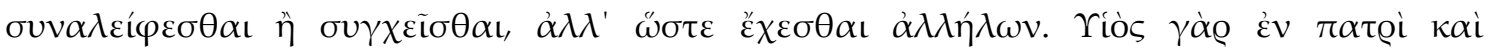

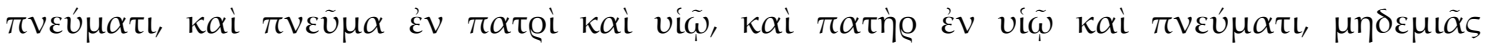

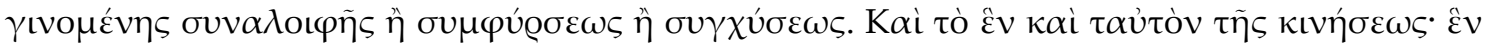

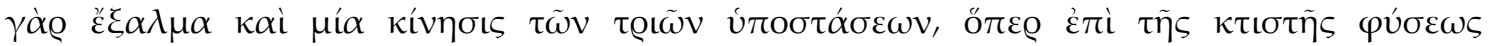

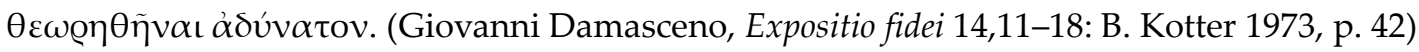

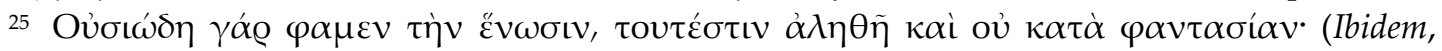
47,66-67: p. 114) 
Il Verbo si unisce alle proprietà della natura umana, infatti queste sono sue perché sono della carne santa, e trasmette le proprietà [divine] alla carne

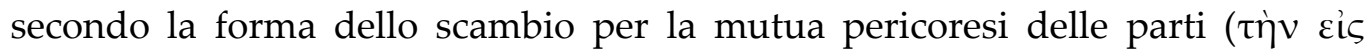
$\not ̋ \lambda \lambda \eta \lambda \alpha \tau \tilde{\omega} \nu \mu \varepsilon \varrho \tilde{\omega} \nu \pi \varepsilon \varrho \iota(\omega \dot{\varrho} \eta \sigma เ v)$ e l'unione secondo l'ipostasi, e poiché uno e il medesimo è colui che secondo ciò che è umano e ciò che è divino "opera secondo la mutua comunione in ciascuna forma" (Leone, Tomus ad Flavianum, $4) .{ }^{26}$

La continuità con la teologia di Massimo è evidente. Significativa è qui pure la citazione del Tomus ad Flavianum. Il Damasceno si inserisce nella teoria delle formulazioni conciliari per farne emergere la consonanza. L'unità nell'ipostasi e la dinamica dello scambio delle proprietà sono, così, espresse con grande chiarezza, senza lasciar spazio alcuno per una confusione dell'aspetto sostanziale con quello personale:

E questa è la forma dello scambio: ciascuna natura trasmette all'altra le proprie caratteristiche attraverso l'identità dell'ipostasi e la reciproca interpenetrazione

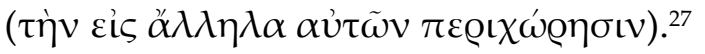

In tal modo il Cristo è nello stesso tempo consustanziale al Padre e allo Spirito secondo la divinità e consustanziale alla madre e a tutti gli uomini secondo l'umanità. ${ }^{28}$ Da qui si vede come a livello ontologico la salvezza può estendersi a tutti gli uomini, proprio quella salvezza che consiste nella partecipazione alla vita divina, cioè alla Vita infinita ed eterna che è la Trinità.

Il n. 49 dell'Expositio fidei articola in modo preciso ed intenzionale in rapida sequenza la dimensione trinitaria, quella cristologica e quella soteriologica. Lo snodo dogmatico fondamentale è il rapporto tra economia ed immanenza, in perfetta consonanza con la teologia cappadoce. Il testo affronta, infatti, prima l'unità trinitaria nell'immanenza divina, per passare poi a quella del Cristo nell'economia e, infine, confrontare le due.

La sequenza del Damasceno è inversa rispetto allo sviluppo storico qui diacronicamente ricostruito. Infatti, il punto di partenza è per lui "sistematicamente" la perichôresis trinitaria, in quanto dice delle Persone divine:

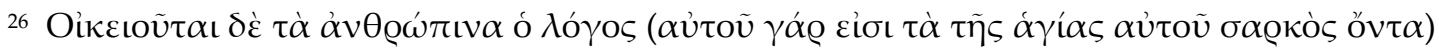

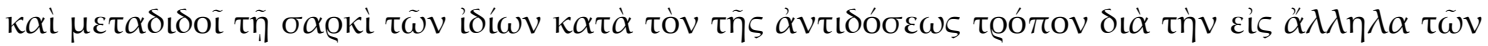

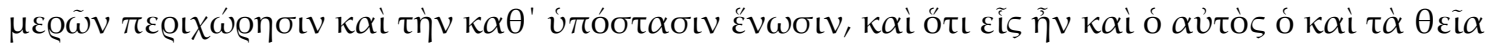

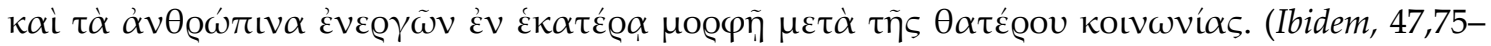
79: p. 115)

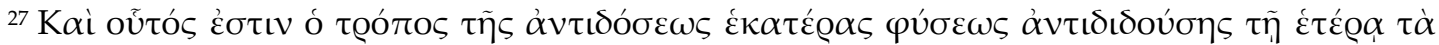

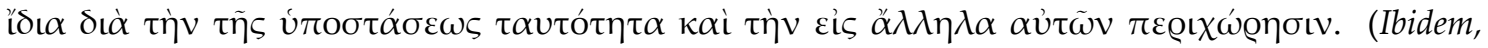
48,38-40: p. 117)

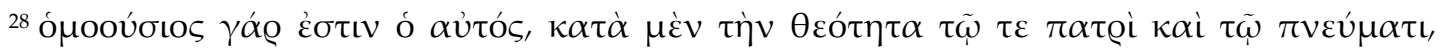

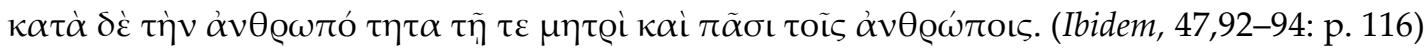




\section{GIULO MASPERO}

E le conosciamo come inseparabili, senza intervallo reciproco, in unità e

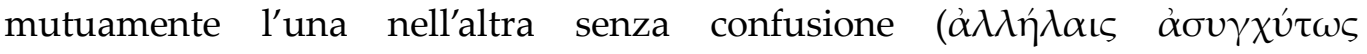
$\pi \varepsilon \varrho \iota \chi \omega \varrho o u ́ \sigma \alpha \varsigma \dot{\varepsilon} \pi \iota \sigma \tau \alpha \dot{\mu} \mu \varepsilon \theta \alpha)$. E sappiamo che sono unite senza confusione ( $\dot{\alpha} \sigma v \gamma \chi \dot{v} \tau \omega \varsigma)$, in quanto sono tre anche se sono in unità, ma possono anche essere riconosciute come distinte senza separazione ( $\dot{\delta} \delta \alpha \sigma \sigma \tau \alpha \dot{\tau} \omega \omega)$ ). Infatti, se ciascuna Persona sussiste in sé ipostaticamente, cioè è perfetta, e come ipostasi possiede veramente la sua caratteristica propria cioè il proprio distinto modo di

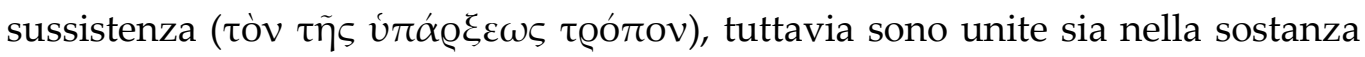
sia nelle proprietà della natura e per il fatto che non si separano né sono divise da un intervallo rispetto all'ipostasi del Padre sono e sono dette essere un unico Dio. $^{29}$

Significativo è il modo escogitato dal Damasceno per presentare contemporaneamente e in forma correlativa le proprietà personali del Padre, del Figlio e dello Spirito, che coincidono con il loro proprio tropos tês yparcheôs, e l'identità di sostanza dalla quale discende l'identità delle proprietà naturali. La sussistenza ipostatica permane dunque inconfusa nella perfetta identità della natura.

Quindi, di seguito, nello stesso n. 49, l'esposizione si sposta dall'immanenza all'economia, per mettere in parallelo la distinzione personale intratrinitaria con la distinzione delle nature nel Cristo:

E allo stesso modo anche a proposito della divina e ineffabile e superiore ad ogni intelletto e comprensione economia dell'unico Verbo divino della Santa Trinità, nostro Signore Gesù Cristo confessiamo sia due nature, quella divina e quella umana, reciprocamente congiunte ( $\sigma v v \varepsilon \lambda \eta \lambda v \theta v i ́ \alpha \varsigma)$ e unite secondo l'ipostasi, sia l'unica ipostasi che sussiste nella sintesi che ha origine dalle due nature. E affermiamo che le due nature sono preservate anche dopo l'unione nell'unica ipostasi che le unisce, cioè nell'unico Cristo, e che veramente di queste nature sono preservate anche le proprietà naturali, unite senza confusione, ma distinguibili e numerabili senza separazione. ${ }^{30}$

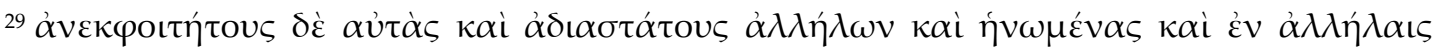

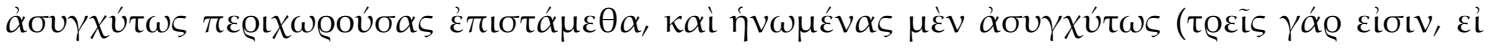

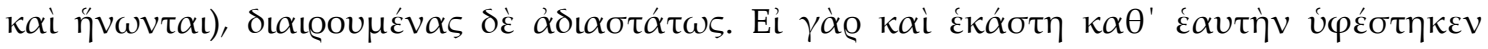

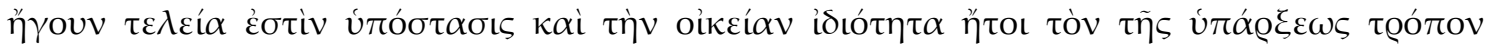

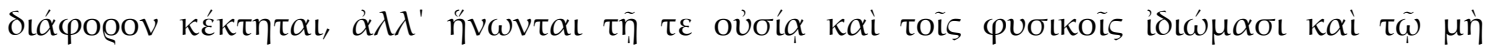

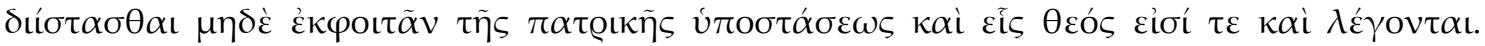
(Ibidem, 49,6-13: p. 118)

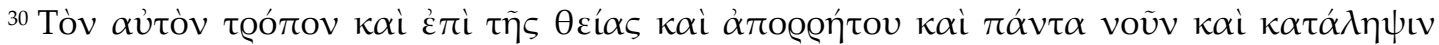

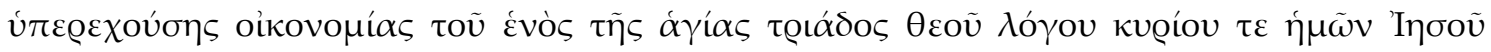

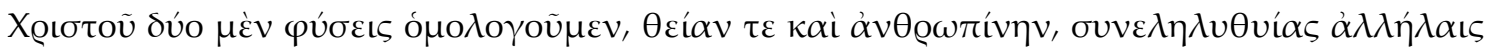

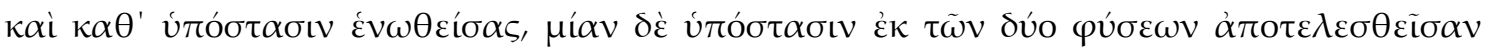

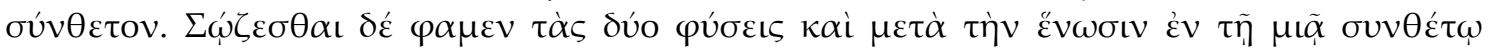


Qui emerge la stessa teologia proposta da Giustiniano nel III Concilio di Costantinopoli, in base alla quale le proprietà naturali permangono distinguibili e numerabili nell'unione senza confusione. Emerge chiaramente il parallelo tra l'unità di natura nella trinità di Persone nell'immanenza divina e l'unità ipostatica nella duplicità di nature nell'economia, cioè nel Cristo.

Lo snodo dogmatico attorno al quale ruota tale corrispondenza è la soteriologia, perché nell'economia salvifica l'unicità della Persona divina fa passare le proprietà naturali da Dio all'uomo, in modo tale da divinizzarlo. Tale parallelismo tra la Trinità e il Cristo è manifestato chiaramente dal ricorso al termine perichôresis anche in questo caso:

E come le tre ipostasi della Santa Trinità sono unite senza confusione e si distinguono e numerano senza separazione, e il numero non produce in loro né differenza, né distanza, né estraniamento, né separazione, poiché riconosciamo che il Padre, il Figlio e lo Spirito Santo sono un unico Dio, allo stesso modo anche le due nature del Cristo, pur essendo unite, sono unite senza confusione, e se inabitano reciprocamente una nell'altra ( $\dot{\varepsilon} v \dot{\alpha} \lambda \lambda \eta \dot{\lambda} \lambda \alpha \iota \varsigma \pi \varepsilon \varrho \iota \chi \omega \varrho \tilde{v} \sigma \iota v),{ }^{31}$ tuttavia non interviene tra loro nessun cambiamento o trasformazione. Infatti, ciascuna natura preserva immutata la propria caratteristica naturale. Per questo è possibile numerarle ma il numero non introduce una differenza. ${ }^{32}$

Immanenza trinitaria, soteriologia e ontologia del Cristo sono perfettamente allineate anche visivamente nel testo del Damasceno. Come è impossibile confondere le tre ipostasi dell'unico Dio in un'unica ipostasi, così è impossibile cancellare la differenza delle nature del Cristo in un'unica natura. ${ }^{33}$ Il parallelismo è intenzionale ed esplicito, e poco oltre se ne spiega la ragione teologica:

Ma si sa che anche se diciamo che le nature del Signore inabitano reciprocamente una nell'altra ( $\pi \varepsilon \varrho \iota \omega \varrho \varepsilon \tilde{\imath} \nu \dot{\varepsilon} \nu \dot{\alpha} \lambda \lambda \eta \dot{\lambda} \alpha \iota \varsigma)$, tuttavia sappiamo che

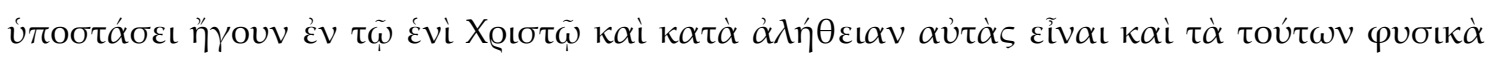

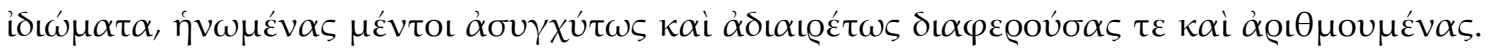
(Ibidem, 49,13-21: p. 118)

${ }^{31}$ La traduzione del verbo con inabitare cerca di rendere anche terminologicamente lo sviluppo teologico, che dalla prima accezione spaziale assume un senso tecnico spirituale.

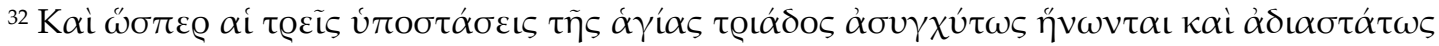

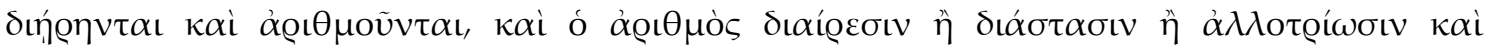

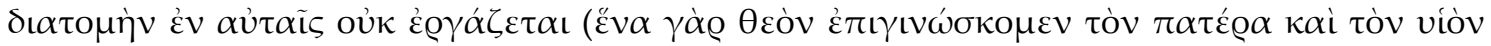

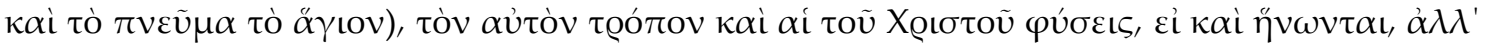

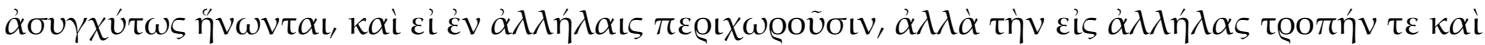

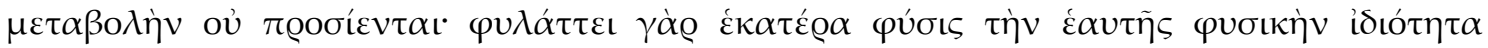

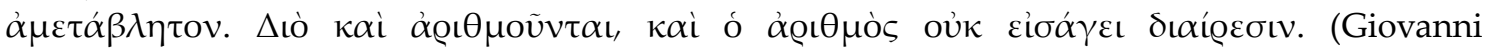
Damasceno, Expositio fidei, 49,21-29: pp. 118-119)

${ }_{33}$ Cf. ibidem, 49,37-42: p. 119. 


\section{GIULO MASPERO}

tale mutua inabitazione ( $\pi \varepsilon \rho \iota \chi \omega ́ \emptyset \eta \sigma \iota)$ deriva dalla natura divina. Infatti essa pervade ogni cosa come vuole e inabita in tutto, ma nulla passa attraverso di essa. ${ }^{34}$

Il passo non può essere sottovalutato, perché qui l'ontologia del Cristo è presentata come riflesso ed espressione dell'ontologia della Trinità. La perichôresis delle Persone divine nell'immanenza è presentata da Giovanni Damasceno come sorgente della perichôresis delle nature a livello cristologico, fondamento della soteriologia e della mistica cristiane. Se la Trinità è Dio, allora il suo essere pericoretico si riflette nella struttura ontologica della salvezza.

Sarà questo principio a fondare il ragionamento in difesa delle icone nel II Concilio di Nicea, dove il ricorso al Damasceno giocò un ruolo fondamentale. La relazionalità delle Persone divine venne, così, letta come sorgente di salvezza nell'unità del Cristo, dal quale sgorga la possibilità di una partecipazione relazionale che passa anche attraverso le immagini, senza che ciò possa ingenerare confusione alcuna tra la trascendenza di Dio e la storia.

\section{Conclusioni}

Alla fine del percorso così tracciato si può costatare come il parallelismo tra il darsi di unità e molteplicità senza né confusione né separazione nell'ontologia del Cristo e quello nella Trinità è presente fin dal pensiero Cappadoce. La terminologia legata alla perichôresis fece il suo ingresso in ambito cristologico grazie a Gregorio di Nazianzo per essere poi sviluppata da Massimo il Confessore in chiave soteriologica. La continuità tra i due Padri è esplicita, insieme alla connessione sistematica tra l'unità cristologica e quella trinitaria, estesa all'ambito delle operazioni. Come nel IV secolo il rapporto tra economia ed immanenza era stato l'asse teologico per la formulazione della distinzione delle Persone divine nell'unità dell'azione, così due secoli dopo la distinzione delle nature si riflette in quella delle operazioni divine e umane del Cristo, che possono essere colte come distinte nella loro unità solo attraverso la theôria, secondo quanto afferma il II Concilio di Costantinopoli. Con Giovanni Damasceno la connessione tra l'unione trinitaria e quella del Cristo sarà presentata con compiutezza proprio attraverso il termine perichôresis, da Lui utilizzato anche per presentare la mutua immanenza delle Persone divine come sorgente della mutua immanenza delle nature del Cristo e, quindi, delle sue operazioni. Da qui discende la divinizzazione dell'uomo, intesa come mutua

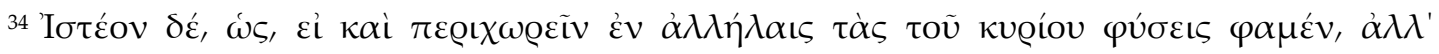

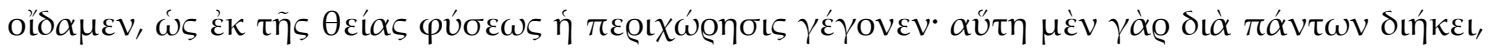

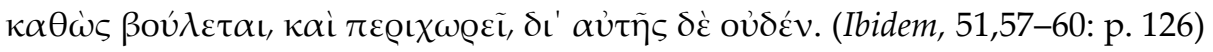


inabitazione delle Persone divine nell'uomo e dell'uomo nella natura del Dio Trinità.

Così, in base alla ricostruzione proposta a partire dal fil rouge dell'uso (chrêsis) del termine perichôresis, si può costatare l'esistenza di un coerente trinitarismo conciliare che di per sé forma un tutt'uno, quasi pericoreticamente, con una cristologia conciliare, nella linea di Timothy Pawl.

A Nicea l'affermazione dell'homousios del Figlio rispetto al Padre aveva aperto la questione fissata poi nel I Concilio di Costantinopoli della relazione tra l'unità trinitaria e quella del Cristo. La connessione ideale tra i due concili, manifestata anche dalla intensità degli eventi che hanno portato dal primo al secondo, rivela come la questione trinitaria e quella cristologica fossero inseparabili, tanto che la risposta all'apollinarismo e quella agli pneumatomachi inevitabilmente si intrecciarono. Il punto era, ovviamente, determinare in quale senso il Padre e il Figlio fossero una cosa sola pur essendo distinti. La questione cristologica qui implicita non poteva essere disgiunta dalla questione dell'unità interna del Cristo e dalla unitrinità di Dio, formulata con forza nel momento dell'affermazione della divinità della terza Persona. I termini ousia, hypostasis e physis furono così risemantizzati e nelle loro relazioni divennero mezzo di espressione del mistero, come vere e proprie icone.

Ma una volta affermata la distinzione tra l'immanenza divina e l'economia della salvezza, rimanevano da approfondire le corrispondenze con il Cristo e con l'umanità. Maria poteva essere veramente definita Madre di Dio? La questione della Theotokos sorgeva, così, come discussione sulla relazionalità dell'homousia del Cristo nei confronti degli uomini rispetto a quella nei confronti del Padre. A Calcedonia la formulazione parallela dell'unità nelle due nature fissò staticamente le corrispondenze, che furono rilette in senso dinamico dai teologi di Giustiniano nel II Concilio di Costantinopoli. La strategia, qui, fu proprio il riportare la compenetrazione delle nature del Cristo a quella delle Persone divine nella Trinità.

La perichôresis, introdotta da Nazianzeno in ambito cristologico, segue tale percorso, tornando in primo piano con Massimo il Confessore per esprimere la dinamicità delle facoltà e delle operazioni insieme alla loro compenetrazione sempre a livello cristologico e soteriologico. Il III Concilio di Costantinopoli poté in tal modo formulare il ditelismo. Infine, nella sintesi del Damasceno, le corrispondenze tra la duplicità delle nature nell'unità dell'ipostasi del Cristo e la trinità delle Persone nell'unità della natura di Dio saranno formulate in tutta la loro compiutezza. Ma proprio tale grammatica teologica, che permetteva di muoversi dall'ontologia della Trinità a quella del Cristo e dell'umanità salvata in Lui, sarà la base del II Concilio di Nicea, il quale chiuderà il "canone" conciliare, in base alla possibilità di risalire relazionalmente dall'icona alla Trinità. 


\section{GIULO MASPERO}

Le discussioni continueranno e la teologia successiva dovette affrontare ulteriori sfide, come un semplice riferimento alla questione del Filioque basterebbe a dimostrare, ma la perichôresis conciliare rimarrà a fissare una methodos, cioè, letteralmente, una possibilità di cammino, per articolare dinamicamente l'unità e la molteplicità, che da Dio si comunica all'uomo nel Cristo. In tale modo l'espressione in esame può essere considerata un magnifico esempio di come l'ontologia trinitaria si sia tradotta in un'antropologia trinitaria nel fondamento dell'epistemologia teologica.

\section{Bibliografia}

Bouchet, Jean-René. 1968. "Le vocabulaire de l'union et du rapport des natures chez S. Grégoire de Nysse." Revue Thomiste 68: 533-82.

Childs, Brevard S. 1979. Introduction to the Old Testament as Scripture. London: SCM Press.

Daley, Brian E. 2018. God Visible: Patristic Christology Reconsidered. Oxford: Oxford University Press. https://doi.org/10.1093/oso/9780199281336.001.0001.

Gnilka, Christian. 2012. Chrêsis: die Methode der Kirchenväter im Umgang mit der antiken Kultur: Der Begriff des "rechten Gebrauchs". Basel: Schwabe.

Grillmeier, Alois. 1990. Jesus der Christus im Glauben der Kirche, I, Freiburg im Breisgau: Herder.

Harrison, Verna. 1991. "Perichoresis in the Greek Fathers." St. Vladimir's Theological Quarterly 35, no. 1: 53-65.

Louth, Andrew. 1996. Maximus the Confessor. London: Routledge.

Mănăstireanu, Dănuț. 2007-2008. "Perichoresis. And the Early Christian Doctrine of God." ARCHÆVVS. Studies in the History of Religions XI-XII: 61-93.

Maspero, Giulio. 2004. "La cristología de Gregorio de Nisa desde la perspectiva del II Concilio de Costantinopla." Scripta Theologica 36: 359-73.

Mateo-Seco, Lucas F. 2003. "Notas sobre el lenguaje cristológico de Gregorio de Nisa." Scripta Theologica 35: 89-112

Moreschini, Claudio. 2000. Gregorio di Nazianzo. Tutte le orazioni. Milano: Bompiani.

Pawl, Timothy. 2016. In Defence of Conciliar Christology. Oxford: Oxford University Press. https://doi.org/10.1093/acprof:oso/9780198765929.001.0001.

Prestige , Leonard. 1928. "Perichoreo and Perichoresis in the Fathers." Journal of Theological Studies 29: 242-52. https://doi.org/10.1093/jts/os-XXIX.115.242.

Raztinger, Joseph. 1971. Introduzione al cristianesimo. Brescia: Queriniana.

Sanders, James A. 1984. Canon and Community: A Guide to Canonical Criticism. Philadelphia (PA): Fortress.

Scalise, Brian T. 2012. "Perichoresis in Gregory of Nazianzen and Maximus the Confessor." Eleutheria 2(1): 58-76. 
Stemmer, Peter. 1983. "Perichorese. Zur Geschichte: eines Begriffs." Archiv für Begriffsgeschichte 27: 9-55.

Stramara, Daniel F. 1998. “Gregory of Nyssa's Terminology for Trinitarian Perichoresis." Vigiliae Christianae 52: 257-63. https://doi.org/10.1163/157007298X00155.

Tixeront, Joseph. 1909. Histoires des dogmes, II, Paris: Gabalda.

Twombly, Charles. 2015. Perichoresis and personhood: God, Christ, and salvation in John of Damascus. Eugene (OR): Pickwick.

Zachhuber, Johannes. 2014. "Individuality and the Theological Debate about Hypostasis." In Individuality in Late Antiquity, edited by Torrance Alexis e Zachhuber Johannes, 91-110. Farnham: Ashgate. https://doi.org/10.4324/9781315588414-6.

Zachhuber, Johannes. 2015. "Derivative Genera in Apollinarius of Laodicea: Some remarks on the philosophical coherence of his thought." In Apollinaris von Laodizäa und die Folgen, edited by Silke-Petra Bergjan et al., 93-113. Tübingen: Mohr Siebeck.

Published Online: April 30, 2020 